\title{
Incidence and risk factors of axial symptoms after cervical disc arthroplasty: a minimum 5-year follow-up study
}

\author{
Jing Chen ${ }^{1,2}$, Jia Li ${ }^{1,2^{*}}$, Gang Qiu ${ }^{3}$, Jingchao Wei ${ }^{3}$, Yanfen Qiu ${ }^{1,2}$, Yonghui An ${ }^{1,2}$ and Yong Shen ${ }^{1,2}$
}

\begin{abstract}
Background: The purpose of this study was to investigate whether uncovertebral joint ossification was a risk factor for axial symptoms (AS) after cervical disc arthroplasty (CDA).

Methods: This retrospective study included 52 consecutive patients who underwent CDA for single-level cervical disc disease. To examine possible risk factors for AS after CDA, univariate and multivariate logistic regression analyses were conducted to compare data from the patients with and without AS (the AS and no-AS groups, respectively).

Results: Among the 52 patients examined, AS were observed in 24 patients $(46.2 \%)$, including a stiff neck $(n=11)$, neck pain and dullness $(n=10)$, and shoulder pain $(n=3)$. Uncovertebral joint ossification was detected in 22 (42.3\%) patients, including 17 patients in the AS group and 5 patients in the no-AS group. Clinical outcome improved during the follow-up period for the AS group. According to multivariate logistic regression analysis, uncovertebral joint ossification, cervical kyphosis, and range of motion (ROM) at the index level were identified as significant risk factors for AS after CDA.

Conclusions: Satisfactory clinical outcomes were observed following CDA for the treatment of single-level cervical disc disease in the present cohort. In addition, uncovertebral joint ossification, cervical kyphosis, and ROM at the index level were found to affect the incidence of AS after CDA.
\end{abstract}

Keywords: Cervical disc arthroplasty, Uncovertebral joint ossification, Cervical kyphosis, Range of motion, Axial symptoms, Clinical outcome

\section{Background}

Cervical disc arthroplasty (CDA) is an alternative to anterior cervical discectomy and fusion (ACDF) for the treatment of single-level cervical spine disease. A theoretical advantage of CDA is its capacity to preserve range of motion (ROM), thereby potentially reducing adjacent level stresses [1-3]. However, after CDA, patients after CDA often complain of neck and/or shoulder pain, a stiff neck, or a dull neck ache. These symptoms are, collectively, referred to as axial symptoms (AS). In previous

\footnotetext{
* Correspondence: ljlyqbwin2010@yeah.net

'Department of Spine Surgery, The Third Hospital of Hebei Medical University, Shijiazhuang 050051, People's Republic of China

${ }^{2}$ The Key Laboratory of Orthopedic Biomechanics of Hebei Province, The Third Hospital of Hebei Medical University, Shijiazhuang 050051, People's Republic of China

Full list of author information is available at the end of the article
}

studies, these symptoms have been attributed to lesions of the disc and facet joints or lesions affecting the muscles of the neck and shoulders [4-6]. In a study by Kawakami et al., a relationship between AS and cervical alignment after ACDF was identified, the authors hypothesized that increased height of the anterior vertebral body affected the incidence of AS after ACDF [7].

Heterotopic ossification ( $\mathrm{HO}$ ) is a common postoperative complication of joint arthroplasty and CDA. Many factors affect the clinical results and development of $\mathrm{HO}$, including gender, advanced age, and multi-level CDA. In addition, $\mathrm{HO}$ has been found to affect ROM at the index level. However, it has not been found to have a negative influence on clinical outcome [8-10]. More recently, Chung et al. demonstrated that uncovertebral 
hypertrophy was a significant risk factor for $\mathrm{HO}$ after CDA [11].

In addition to these risk factors, we propose that uncovertebral joint ossification may represent another risk factor for AS after CDA. Uncovertebral joint ossification has the potential to alter dynamics at the index level and may also affect clinical outcome, adversely. Therefore, the purpose of this study was to investigate whether uncovertebral joint ossification was a risk factor for AS after CDA.

\section{Methods}

This retrospective study included a total of 52 patients who underwent single-level CDA in the Third Hospital of Hebei Medical University between July 2004 and June 2009. Inclusion criteria were myelopathy and/or radiculopathy from single-level disc herniation in adult patients that was nonresponsive to appropriate nonsurgical treatment for at least 3 months. Patients with previous cervical spine surgery, an active infection, uncovertebral joint ossification, severe spondylosis and/or disc height loss, ossification of the posterior longitudinal ligament, or kyphotic deformity were excluded from this study. This study was approved by the Regional Ethics Committee of The Third Hospital of Hebei Medical University, and all patients signed informed consent forms.

\section{Surgical technique}

For each patient, CDA was performed by the same senior surgeon. Briefly, an anterior approach via a rightside skin incision was used to perform the surgical procedures. The posterior longitudinal ligament was completely excised and the spinal canal and neuroforamen were decompressed. Endplates were prepared according to the Bryan disc milling technique and this created two concave surfaces. The operative site was then routinely irrigated with saline prior to insertion of the devices.
Prior to surgery, the appropriate BRYAN cervical disc (Medtronic Sofamor Danek) was selected based on templating and radiographic studies that included computed tomography to assure appropriate placement.

\section{Evaluation criteria}

Clinical evaluations, including radiological and clinical evaluation results, were collected preoperatively and also at each follow-up. When the follow-up period was longer than 5 years, the last set of available data was used for statistical analysis. The modified Japanese Orthopedic Association (JOA) scoring system was used to determine functional status before surgery and at the final follow-up visit. Both the neck disability index (NDI) and visual analog scale (VAS) were used to evaluate neck and arm pain. AS included neck and/or shoulder pain, a stiff neck, or a dull neck ache. Also at the last follow-up, patients were divided into an AS group or a no-AS group according to whether or not they were experiencing AS.

The radiographic evaluation performed included computed tomography and static and dynamic flexion/extension lateral images. The presence of uncovertebral joint ossification was assessed in these images by two independent doctors who were blinded to the clinical outcome of each case (Fig. 1). HO was also assessed from the dynamic flexion/extension lateral images and was graded as described by McAfee et al. [12]. Disc height was defined based on the average heights of the anterior and posterior discs. ROM at the index level was determined by drawing lines between the superior endplate of the adjacent cephalad vertebral body and the inferior endplate of the adjacent caudal vertebral body (Fig. 2). The functional spinal unit (FSU) angle was examined on lateral radiographs and was determined based on the lines drawn at the superior end plate of the cephalad vertebral body and at the inferior end plate of the caudal vertebral body (Fig. 2).

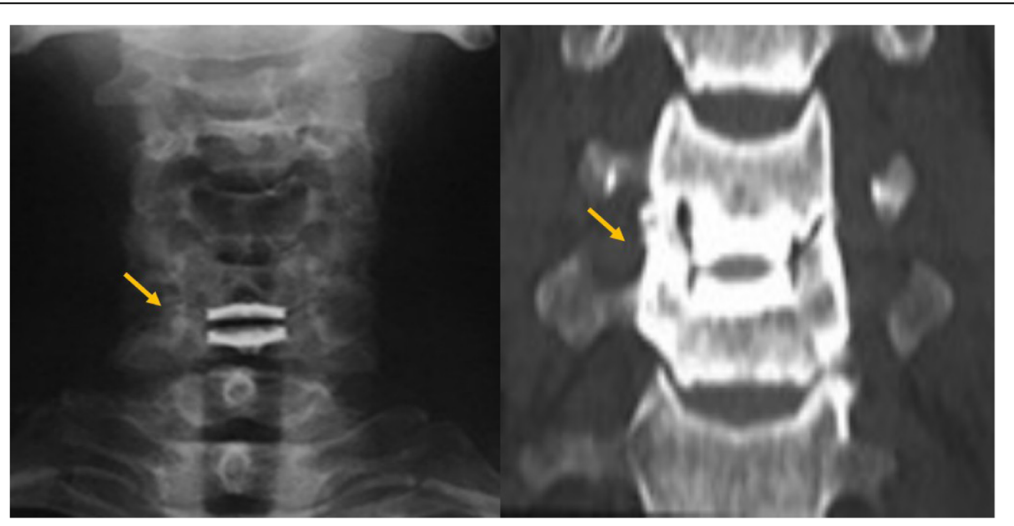

Fig. 1 Uncovertebral joint ossification is observed in anteroposterior views from computed tomography and X-ray images 


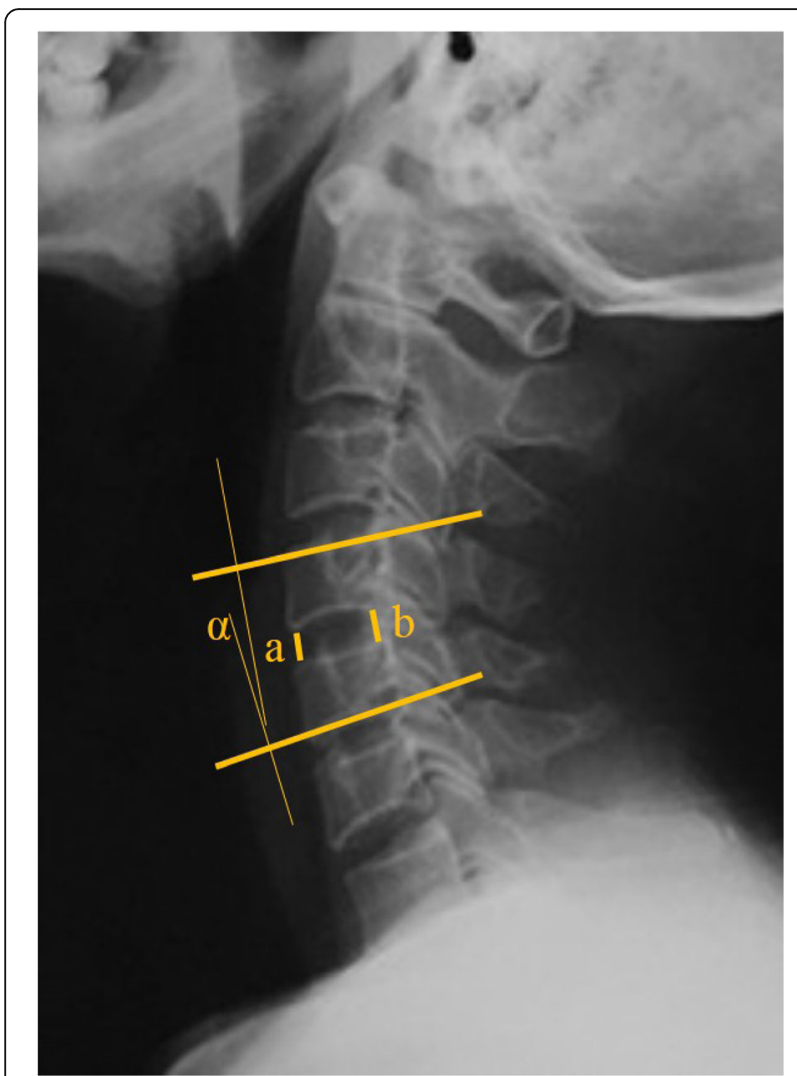

Fig. 2 The functional spinal unit angle $(a)$ was examined on lateral radiographs and was formed by lines drawn at the superior end plate of the cephalad vertebral body and at the inferior end plate of the caudal body. Disc height was defined based on the average values of the anterior disc height $(a)$ and the posterior disc height (b)

\section{Statistical analysis}

Statistical analyses were performed using SPSS software (version 17.0, Chicago, IL, USA). Significant differences between preoperative and final follow-up measurements were identified by using a paired sample $t$ test. An independent $t$ test or Chi-square test was used to identify significant differences between groups. Multivariate logistic regression analysis was used to identify risk factors related to the incidence of AS. In all analyses performed, significance was defined as a $P$ value less than 0.05 . Results are presented as the mean \pm standard deviation.

\section{Results}

A total of 52 patients were included in this study and their mean follow-up period was $5.7 \pm 0.6$ years. AS were observed in 24 patients (46.2\%), and these included a stiff neck $(n=11)$, neck pain and dullness $(n=10)$, and shoulder pain $(n=3)$. None of the patients required additional surgery on either the index level or adjacent levels for recurrent symptoms. HO was observed in 27/ 52 (51.9\%) patients. Among these patients, 24 patients were classified as grade $1 / 2$, while 3 patients were classified as grade 3 . Grade 4 , which has the potential to develop into complete arthrodesis, was not observed in the present cohort. Uncovertebral joint ossification was observed in 22/52 (42.3\%) patients of the cohort, with 17 of these patients in the AS group and 5 of these patients in the no-AS group $(P<0.05)$ (Table 1$)$.

In both groups, the JOA, NDI, and VAS scores for neck pain and arm pain were significantly improved in the follow-up period compared to the preoperative scores $(P<0.05)$. Furthermore, except for neck pain $(P<0.05)$, there were no significant differences in these scores between the two groups at the final follow-up $(P>0.05)$. The mean postoperative disc height was $8.2 \pm 1.1 \mathrm{~mm}$ in the AS group and $7.9 \pm 1.2 \mathrm{~mm}$ in the no-AS group, and this difference was not significant. In contrast, ROM at the index level for the AS and no-AS groups were $6.3 \pm 1.8^{\circ}$ and $8.8 \pm 2.7^{\circ}$, respectively, and this difference was significant $(P<0.05)$. The FSU angles for the AS and no-AS groups were $-0.1 \pm 5.2^{\circ}$ and $5.0 \pm 3.9^{\circ}$, respectively, at the final follow-up, and these differences were also significant $(P<0.05)$ (Table 2$)$.

To compare the relative impact of these variables on the incidence of AS, multiple logistic regression analysis was performed. With a $P$ value $<0.1$ applied in a univariate analysis, ROM at the index level, uncovertebral joint ossification, and FSU angle were analyzed as dependent variables with a forward stepwise method. Based on this analysis, uncovertebral joint ossification, cervical kyphosis, and ROM at the index level were identified as significant risk factors for AS after CDA (Table 3).

\section{Discussion}

The results of this study demonstrated that CDA achieved a good clinical outcome, with the overall incidence of uncovertebral joint ossification following CDA being $42.3 \%$. Furthermore, significant risk factors identified for AS following arthroplasty included uncovertebral joint ossification, cervical kyphosis, and ROM at the index level. CDA is designed to preserve cervical motion and decrease the incidence of adjacent segment degeneration

Table 1 Demographics of the cohort examined

\begin{tabular}{llll}
\hline Parameter & AS group & No-AS group & $P$ value \\
\hline Age (years) & $46.9 \pm 5.3$ & $44.3 \pm 6.2$ & 0.571 \\
Gender (male/female) & $11 / 13$ & $12 / 16$ & 0.829 \\
Operated segment & & & 0.960 \\
C4/C5 & 5 & 5 & \\
C5/C6 & 11 & 13 & \\
C6/C7 & 8 & 10 & 0.163 \\
Operation time (min) & $70.3 \pm 9.6$ & $68.5 \pm 11.3$ & 0.112 \\
Follow-up (years) & $5.9 \pm 0.6$ & $5.6 \pm 0.3$ & \\
\hline
\end{tabular}


Table 2 Comparisons of outcome factors examined preoperatively versus postoperatively within groups and between the AS and No-AS groups within time points

\begin{tabular}{llllll}
\hline & \multicolumn{2}{l}{ AS group } & & \multicolumn{2}{l}{ No-AS group } \\
\cline { 2 - 3 } Outcome & Preoperative & Last follow-up & & Preoperative & Last follow-up \\
\hline JOA & $9.8 \pm 2.2$ & $14.6 \pm 1.6^{*}$ & & $10.1 \pm 3.6$ & $14.9 \pm 2.9^{*}$ \\
NDI & $46.3 \pm 7.2$ & $19.6 \pm 3.1^{*}$ & & $45.3 \pm 5.9$ & $16.1 \pm 2.3^{*}$ \\
VAS for & $6.9 \pm 3.5$ & $3.3 \pm 1.2^{*, * *}$ & $6.7 \pm 2.8$ & $1.8 \pm 0.9^{*, * *}$ \\
neck pain & & & & \\
VAS for & $6.3 \pm 2.1$ & $1.6 \pm 0.7^{*}$ & & $6.5 \pm 2.1$ & $1.3 \pm 0.9^{*}$ \\
arm pain & & & & \\
ROM & $7.2 \pm 2.1^{\circ}$ & $6.3 \pm 1.8^{* *}$ & & $7.9 \pm 1.8^{\circ}$ & $8.8 \pm 2.7^{* *}$ \\
FSU angle & $0.6 \pm 0.3^{\circ}$ & $-0.1 \pm 5.2^{* * * *}$ & & $0.7 \pm 0.2^{\circ}$ & $5.0 \pm 3.9^{* * * *}$ \\
Disc height & $7.0 \pm 1.3$ & $8.2 \pm 1.1^{*}$ & & $7.1 \pm 0.9$ & $7.9 \pm 1.2^{*}$ \\
\hline
\end{tabular}

*There was a significant difference between baseline and final follow-up; $P<0.05$

**There was a significant difference between the AS and no-AS groups; $P<0.05$

${ }^{\circ}$ means degree

[13]. Occurrence of $\mathrm{HO}$ is a common postoperative complication after CDA and has the potential to limit the motion of artificial disc prostheses. When Yi et al. [10] analyzed 170 patients after CDA, with the duration of follow-up being longer than 1 year, the incidence of $\mathrm{HO}$ was $40.6 \%$. Similarly, Lee et al. found that $27.1 \%$ of patients developed $\mathrm{HO}$ after a follow-up period of 14 months [14]. More recently, Zhao et al. [15] reported an incidence rate of $69.0 \%$ for $\mathrm{HO}$ over a follow-up period of 10 years for a Chinese population. In the present study, $51.9 \%$ of all the patients and operated segments in our series had radiographic evidence of grades 1 or $2 \mathrm{HO}$ at their last follow-up, and $42.3 \%$ of the patients exhibited uncovertebral joint ossification. Van Ooij et al. [16] previously demonstrated that an abnormal movement pattern of segments with a disc prosthesis could explain the incidence of $\mathrm{HO}$. In the present series, postoperative ROM and FSU angles in the AS group significantly differed from the noAS group $(P<0.05)$. We hypothesize that the cause of $\mathrm{HO}$ was abnormal movements in response to segmental cervical kyphosis, and similar outcomes have been observed with lumbar disc arthroplasty [16].

Several studies have reported the influence of gender, age, and multi-level and uncovertebral hypertrophy on $\mathrm{HO}$ after CDA $[8,10,11]$. However, it remains unclear whether these factors affect the incidence of AS after

Table 3 Multivariate analysis of the risk factors for AS

\begin{tabular}{lcll}
\hline Risk factor & $P$ value & OR & $95 \% \mathrm{Cl}$ \\
\hline ROM & 0.027 & 1.761 & $1.1433-2.7144$ \\
FSU angle & 0.003 & 1.977 & $1.3152-2.9719$ \\
Uncovertebral joint ossification & 0.012 & 2.437 & $1.6441-3.6117$ \\
\hline
\end{tabular}

$O R$ odds ratio, $\mathrm{Cl}$ confidence interval
CDA. Ebraheim et al. [17] showed that uncovertebral joint osteophytes may cause foraminal stenosis and nerve root compression. In a study by Chung et al. [11], preoperative uncovertebral joint hypertrophy was associated with the occurrence of ROM-affecting HO. Therefore, the authors recommended that uncovertebral joint hypertrophy should be assessed before CDA. Quan et al. [18] observed a trend where patients who developed more extensive $\mathrm{HO}$ experienced slightly greater neck pain and had higher arm pain analog scores than patients without HO. However, this difference was not statistically significant. In the present study, ROM at the index level in the AS group was significantly lower compared to the no-AS group $(P<0.05)$. Based on our own experience, a hyperplastic or ruptured posterior longitudinal ligament and anterior and posterior osteophytes of the vertebral body should be removed completely during decompression. In the present study, the endplates were prepared with the Bryan disc milling technique to create two concave surfaces. At the end of the milling process, the resulting outer ridge of bone was able to capture the rim of each shell of the arthroplasty, thereby providing immediate stability. The cortical endplates of the vertebral body also needed to be preserved in order to decrease the risk of subsidence and loosening. Thus, overmilling at the dorsal endplate, the angle of Bryan disc insertion, and loss of lordosis in the disc may contribute to the presence of AS.

After CDA, it is possible that uncovertebral joint ossification accelerates the degeneration of articular surfaces and leads to the growth of osteophytes. This could further hinder ROM, while the development of osteophytes could impinge on nerve roots during movement of the cervical spine. Accordingly, a higher occurrence rate for AS may be related to uncovertebral joint ossification and decreased ROM. In the present study, the AS reported included neck and/or shoulder pain, a stiff neck, or dull neck ache. It remains unclear whether the uncovertebral joint ossification observed will continue to worsen. It is also possible that the biomechanical changes caused by uncovertebral joint ossification could be a contributing factor to AS after CDA.

Previous studies have reported that FSU kyphosis occurs after CDA, and cervical kyphosis was one of the risk factors found to be significantly related to AS after anterior cervical surgery. Correspondingly, Pickett et al. [19] described a patient with postoperative kyphosis that experienced AS and Harrison et al. [20] showed a relationship between cervical kyphosis and AS. In the present study, over-milling of the endplate and asymmetric millings were avoided by selecting an insertion angle parallel to the angle of the native disc space. The structural absence of lordosis in the Bryan prosthesis was a potential cause of segmental cervical kyphosis, 
and therefore, the posterior longitudinal ligament was removed. Based on our experience, it is hypothesized that there are two main contributing factors to cervical kyphosis, over-milling of the endplate and asymmetric millings. Furthermore, the results of the present study also demonstrated a correlation between segmental cervical kyphosis after CDA and AS.

There were limitations associated with our study. First, the incidence of uncovertebral joint ossification and cervical kyphosis after CDA were reported, although there are many factors after CDA that can affect the incidence of AS. It remains to be investigated whether the impact of uncovertebral joint ossification and cervical kyphosis extends to other artificial disc prostheses. The second limitation of our study was the relatively small patient group size. As a result, identification of the precise incidence and risk factors of AS was limited. Moreover, the degree of uncovertebral joint ossification was not evaluated, and it remains to be determined if it correlates with AS. It is also unclear whether uncovertebral joint ossification worsens with time. None of the present cohort needed additional surgery on either the index level or adjacent levels for recurrent symptoms. In future studies, we will evaluate whether, and to what extent, degradation of the adjacent segment occurs. Biomechanical studies on the occurrence of AS in relation to the degenerative process after arthroplasty would also be essential in identifying the pathogenesis of AS. To establish the exact cause(s) of AS, a larger group of patients treated for CDA, including those undergoing a multisegment operative technique, are required. In particular, a prospective multi-center study with long-term followup would provide very useful information.

\section{Conclusions}

The clinical outcomes of CDA for the surgical treatment of single-level cervical disc disease in this cohort were satisfactory. Furthermore, multivariate logistic regression analysis identified uncovertebral joint ossification, cervical kyphosis, and ROM at the index level to be factors that affect the incidence of AS after CDA.

\section{Abbreviations \\ ACDF: Anterior cervical discectomy and fusion; AS: Axial symptoms; CDA: Cervical disc arthroplasty; FSU: Functional spinal unit; HO: Heterotopic ossification; JOA: Japanese Orthopedic Association; NDI: Neck disability index;} ROM: Range of motion; VAS: Visual analog scale

\section{Acknowledgements}

We thank Dr. Yingze Zhang for his support in obtaining the approval of the ethics committee for this study.

\section{Funding}

Not applicable.

Availability of data and materials

Not applicable.

\section{Authors' contributions}

$J$ was responsible for the conception and design of this study, as well as the acquisition and interpretation of data and preparation of a manuscript draft. JC, GQ, JW, and FL contributed to the imaging analysis and the acquisition and interpretation of data. YA, YQ, and YS critically revised the intellectual content of the manuscript and prepared the final version of the manuscript to be published. All of the authors have read and approved the final manuscript.

\section{Competing interests}

The authors declare that they have no competing interests.

Consent for publication

Not applicable.

Ethics approval and consent to participate

This study was approved by the Regional Ethics Committee of The Third Hospital of Hebei Medical University and all patients signed informed consents.

\section{Author details}

'Department of Spine Surgery, The Third Hospital of Hebei Medical University, Shijiazhuang 050051, People's Republic of China. ${ }^{2}$ The Key Laboratory of Orthopedic Biomechanics of Hebei Province, The Third Hospital of Hebei Medical University, Shijiazhuang 050051, People's Republic of China. ${ }^{3}$ Department of Orthopedic Surgery, Hebei General Hospital, 348 Heping Road, Shijiazhuang 050000, People's Republic of China.

Received: 19 April 2016 Accepted: 8 September 2016

Published online: 20 September 2016

\section{References}

1. Zigler JE, Delamarter R, Murrey D, Spivak J, Janssen M. ProDisc-C and anterior cervical discectomy and fusion as surgical treatment for single-level cervical symptomatic degenerative disc disease: five-year results of a Food and Drug Administration study. Spine (Phila Pa 1976). 2013;38(3):203-9.

2. Sasso RC, Anderson PA, Riew KD, Heller JG. Results of cervical arthroplasty compared with anterior discectomy and fusion: four-year clinical outcomes in a prospective, randomized controlled trial. J Bone Joint Surg Am. 2011;93(18):1684-92.

3. Ren $C$, Song $Y$, Xue $Y$, Yang $X$. Mid- to long-term outcomes after cervical disc arthroplasty compared with anterior discectomy and fusion: a systematic review and meta-analysis of randomized controlled trials. Eur Spine J. 2014;23(5):1115-23.

4. Hosono N, Yonenobu K, Ono K. Neck and shoulder pain after laminoplasty. A noticeable complication. Spine (Phila Pa 1976). 1996;21(17):1969-73.

5. Ebata S, Sato H, Ohba T, Ando T, Haro H. Postoperative intervertebral stabilizing effect after cervical laminoplasty. J Back Musculoskelet Rehabil. 2015;28(2):303-9.

6. Ohnari H, Sasai K, Akagi S, lida H, Takanori S, Kato I. Investigation of axial symptoms after cervical laminoplasty, using questionnaire survey. Spine J. 2006;6(3):221-7.

7. Kawakami M, Tamaki T, Yoshida M, Hayashi N, Ando M, Yamada H. Axial symptoms and cervical alignments after cervical anterior spinal fusion for patients with cervical myelopathy. J Spinal Disord. 1999;12(1):50-6.

8. Lee JH, Jung TG, Kim HS, Jang JS, Lee SH. Analysis of the incidence and clinical effect of the heterotopic ossification in a single-level cervical artificial disc replacement. Spine J. 2010;10(8):676-82.

9. Walraevens J, Demaerel P, Suetens P, Van Calenbergh F, van Loon J, Vander Sloten J, Goffin J. Longitudinal prospective long-term radiographic follow-up after treatment of single-level cervical disk disease with the Bryan Cervical Disc. Neurosurgery. 2010;67(3):679-87.

10. Yi S, Shin DA, Kim KN, Choi G, Shin HC, Kim KS, Yoon DH. The predisposing factors for the heterotopic ossification after cervical artificial disc replacement. Spine J. 2013;13(9):1048-54.

11. Chung SB, Muradov JM, Lee SH, Eoh W, Kim ES. Uncovertebral hypertrophy is a significant risk factor for the occurrence of heterotopic ossification after cervical disc replacement: survivorship analysis of Bryan disc for single-level cervical arthroplasty. Acta Neurochir (Wien). 2012;154(6):1017-22. 
12. McAfee PC, Cunningham BW, Devine J, Williams E, Yu-Yahiro J. Classification of heterotopic ossification (HO) in artificial disk replacement. J Spinal Disord Tech. 2003;16(4):384-9.

13. Cao JM, Zhang YZ, Shen Y, XU JX, Ding WY, Yang DL, Zhang D. Clinical and radiological outcomes of modified techniques in Bryan cervical disc arthroplasty. J Clin Neurosci. 2011;18(10):1308-12.

14. Lee DH, Lee JS, Yi JS, Cho W, Zebala LP, Riew KD. Anterior cervical plating technique to prevent adjacent-level ossification development. Spine J. 2013;13(7):823-9.

15. Yanbin Z, Yilong Z, Yu S, Feifei Z, Zhongjun L. Application of cervical arthroplasty with Bryan cervical disc: 10 years follow-up results in China. Spine (Phila Pa 1976). 2015. [Epub ahead of print].

16. Van Ooij A, Oner FC, Verbout AJ. Complications of artificial disc replacement: a report of 27 patients with the SB Charite' disc. J Spinal Disord. 2003;16(4):369-83.

17. Ebraheim NA, Lu J, Biyani A, Brown JA, Yeasting RA. Anatomic considerations for uncovertebral involvement in cervical spondylosis. Clin Orthop Relat Res. 1997;334:200-6.

18. Quan GM, Vital JM, Hansen S, Pointillart V. Eight-year clinical and radiological follow-up of the Bryan cervical disc arthroplasty. Spine (Phila Pa 1976). 2011;36(8):639-46.

19. Pickett $G E$, Mitsis DK, Sekhon $L H$, et al. Effects of a cervical disc prosthesis on segmental and cervical spine alignment. Neurosurg Focus. 2004;17:E5.

20. Harrison DD, Harrison DE, Janik TJ, et al. Modeling of the sagittal cervical spine as a method to discriminate hypolordosis: results of elliptical and circular modeling in 72 asymptomatic subjects, 52 acute neck pain subjects, and 70 chronic neck pain subjects. Spine. 2004;29:2485-92.

\section{Submit your next manuscript to BioMed Central and we will help you at every step:}

- We accept pre-submission inquiries

- Our selector tool helps you to find the most relevant journal

- We provide round the clock customer support

- Convenient online submission

- Thorough peer review

- Inclusion in PubMed and all major indexing services

- Maximum visibility for your research

Submit your manuscript at www.biomedcentral.com/submit

C) Biomed Central 